\section{6 THE PREVALENCE OF AORTIC ROOT DILATION IN
HIGHLY TRAINED ATHLETES: 'THE BRITISH
EXPERIENCE'}

S Gati, ${ }^{1}$ C Sedgwick, ${ }^{2}$ M Papadakis, ${ }^{2}$ M Reed, ${ }^{2}$ A Zaidi, ${ }^{2}$ N Sheikh, ${ }^{2}$ A Cox, ${ }^{2}$ S Ghani, ${ }^{2}$ A Child, ${ }^{2}$ S Sharma ${ }^{1}$ St. George's University of London; ${ }^{2}$ St. George's University

doi:10.1136/heartjnl-2013-304019.106

Introduction Studies suggest there is an increased prevalence of aortic root dilation highly trained competitive athletes. It is unclear whether this is a benign feature of 'athlete's heart' or an indication of a pathological condition. However, distinguishing between the two may be important in the prevention of exercise related sudden cardiac death. This study sought to investigate the prevalence of aortic root dilation in British competitive athletes.

Methods Between 2004 and 2012, 1165 athletes (63\% male, 81\% Caucasian, mean age 20.9 \pm 6.0 years, range $14-35$ years) underwent echocardiography. Aortic root dimensions, measured at sinus of valsalva level, were analysed to ESC guidelines and were compared to current Bathesda recommendations of $\geq 40 \mathrm{~mm}$ for both male and female athletes. Results were compared to 415 healthy controls $(57 \%$ male, $60 \%$ Caucasian, mean age 22.1 \pm 6.4 years, range $14-35$ years) and 50 patients with Marfan syndrome $(54 \%$ male, $88 \%$ Caucasian, mean age $35.6 \pm 12.6$ years, range 18-68 years).

Results Male athletes and controls demonstrated a similar prevalence of aortic root dimension $(27.9 \pm 4.2 \mathrm{~mm}$, range: $17-52 \mathrm{~mm}$ vs $27.9 \pm 4.0 \mathrm{~mm}$, range: 19 "C $38 \mathrm{~mm} ; \mathrm{p}=1.000)$. However, seven male athletes $(1.0 \%)$ had an aortic root $\geq 40 \mathrm{~mm}$, compared to $0 \%$ male controls ( $p$ 0.2053). Male Marfan patients demonstrated a higher prevalence of aortic root dilation than male athletes $(40.9 \mathrm{~mm} \pm 4.6 \mathrm{~mm} ; \mathrm{p}<0.0001)$ and 22 male Marfan patients $(81.5 \%)$ had an aortic root $\geq 40 \mathrm{~mm}$.

Female athletes demonstrated a similar prevalence of aortic root dimension to female controls $(26.1 \pm 3.3 \mathrm{~mm}$ vs $25.8 \pm 3.6$; p 03207). One female athlete $(0.2 \%)$ had an aortic root $\geq 40 \mathrm{~mm}$ compared to $0 \%$ female controls $(p=1.000)$. Female Marfan patients had a higher prevalence of aortic root dilation than female athletes $(36.8$ $\pm 4.7 \mathrm{~mm}$ vs $26.1 \pm 3.3 \mathrm{~mm} ; \mathrm{p}<0.0001)$ and 17 female Marfan patients $(73.9 \%)$ had an aortic root $\geq 34 \mathrm{~mm}$.

On longitudinal follow up of 5 years, none of the athletes with aortic dilation alone required surgical intervention.

Conclusions Athletes exhibited a similar prevalence of aortic root dilation to healthy controls and there is no statistically significant evidence to suggest aortic root dilation is a feature of 'athlete's heart'. However, Marfan's patients were found to have a far greater proportion of pathologically wide aortic of $\geq 40 \mathrm{~mm}$. The study would benefit from further longitudinal evaluation. 